\title{
Why Advanced Alien Civilizations Might Not Communicate the Way We Expect
}

\author{
Andy E. Williams, Nobeah Foundation, Nairobi, Kenya, awilliams@nobeahfoundation.org
}

\section{Keywords}

Kardashev Scale; General Collective Intelligence, Artificial General Intelligence, SETI

\begin{abstract}
General Collective Intelligence or GCI has been defined as a system that orchestrates individuals into a single collective intelligence with the potential for vastly greater problem-solving ability than any individual in the group. Firstly, in that case of any civilization that has achieved GCI, due to its complexity, any transmission for purposes of communication might be perceived as noise. Furthermore GCI is a pattern that might potentially repeat at any order $\mathrm{N}$, resulting in successively more complex communication at successively greater scale. The need to avoid interference in such communication at any given order of GCI suggests such communication on a planetary level within a given civilization might be likely to be wired and not wireless. Finally, identifying and detecting possible modes of communication and deciphering communication between civilizations that have achieved GCI is potentially a problem of higher order complexity that is not reliably solvable without GCI or without another higher order intelligence such as an AGI. Since neither the problem nor the solution can reliably be resolved, by definition higher order problems are those in which one is likely to be trying to solve the wrong problem with the wrong solution. If so, then in trying to detect communication within or between civilizations that have reached the state of implementing a first order or higher order GCI, current efforts in the search for extraterrestrial intelligence (SETI) are likely to be looking in the entirely wrong direction.
\end{abstract}

\section{Introduction}

Estimates for the number of alien civilizations given by the Drake equation, and the use of the Kardashev scale to predict the signatures of alien civilizations, have still so far not resulted in SETI having found unambiguous signs of sentient life elsewhere, even our own galaxy. However a functional model of cognition suggested to be complete enough to represent all the functionality required for human-like general problem solving ability has recently been proposed that might shed some light on this problem. This model represents human cognition as a system that moves though a space of concepts or a "conceptual space", and also suggests that the visual representation of cognition in this conceptual space might show human cognition as a well-defined phase change in animal intelligence at which general problem-solving ability increases exponentially [1]. In this conceptual space a concept is a point, and a generalization is a region containing many points. This phase change, which is suggested to occur when the ability to generalize reaches the point at which reasoning can remain within the same generalization, appears to be a Nth order pattern, in which each increase in order represents an exponential increase in intelligence, and where each order potentially corresponds to a level on the Kardashev scale. In this progression, firstly animal intelligence becomes human intelligence. Then human intelligences organize themselves into a General Collective Intelligence or GCI. Then GCIs organize themselves into a GCI of GCI's, or a second order GCI. And so on.

In this progression, human language is required in order for human intelligence to gain the capacity to achieve an exponential increase in general problem-solving ability over animals. Value in the abstract is impact on any general problem. Therefore the ability to abstract value represents a jump in problem solving ability. Where animals might achieve significant differences in resources from others in their species, through the exchange of specific concepts having value in solving any given problem, in return for something representing value in the abstract, humans can achieve exponentially greater differences 
in resources than any single human is capable of through their individual efforts. Also, problem-solving ability in this functional model roughly equates to the volume of conceptual space that can be navigated per unit time. In order to display this problem-solving ability the cognitive system must have accumulated a sufficient number of concepts (the conceptual space must be large enough) to demonstrate this ability to navigate

Human language structured with functional modeling is required for GCI to gain the capacity to achieve an exponential increase in general problem-solving ability over humans. Similarly each Nth order GCI appears to require it's own language for communication at the level of that GCI. This pattern appears to lead to the capacity to exchange and integrate larger and larger sets of information. Typical interaction patterns in both animals and humans involve an initial heavy uptake of information during the stage in which young animals or humans are learning about the world, followed by more sparse updates on increasingly more specific topics as animals or humans get older. If this pattern repeats at the Nth order, it could potentially result in information being exchanged in communication that is less and less frequent, potentially resulting in higher order civilizations simply exchanging entire regions in cognitive space representing their entire histories and their entire understanding of this existence.

However, in this functional modeling approach the level of enlightenment of an individual or group consciousness is roughly the capacity of the consciousness to navigate the space of awarenesses (the volume of "awareness space" that can be navigated per unit time) to solve the problem of achieving fitness of the consciousness to function (conscious well-being). In this functional modeling approach the goal of any Nth order intelligence, and therefore its goal in any action, including communication, is to increase its well being. But at the same time, as any Nth order intelligence increases its level of enlightenment, there might be less and less information to exchange in order to achieve that. As an example, two individuals with a low level of enlightenment might have to exchange their entire life histories in order to communicate the information required for each to achieve their well-being. More enlightened individuals might merely need a single conversation. At a larger scale, much information that might be communicated between planetary or galactic civilizations might potentially be redundant. If there are common patterns of evolution and development which a higher order GCI might make clear, then all that need be communicated are the differences in the implementations of those patterns, not the redundant information.

Once GCI is developed, individuals of each civilization will always need to interact with the GCI. However the combination of AGI and GCI might potentially use such patterns in the information being communicated to remove the need for a great deal of communication.

Some rough estimations about the nature and parties involved in communication within and between higher order GCI civilizations might be made. Assume that human speech has a universal transmission rate, which has recently been suggested to be 39 bits per second. And assume that for any given level of technology there is a maximum transmission rate for known mechanisms of communication transmission based on electromagnetic radiation, as suggested by the new record for the fastest ever data transmission rate between a single transmitter and receiver that has been set by researchers in the UK, who achieved a rate of 1.125 terabits (approximately $10^{5}$ times the rate of human speech) per second using an optical communications system.

In a first order GCI containing $\mathrm{N}$ individuals, each individual might interact with the GCI through an intelligent agent operating on their behalf (i.e. their own AGI). These AGI might interact with the GCI at orders of magnitude greater speed and scale, up to the maximum transmission rate (again 
approximately $10^{5}$ times the rate of human speech). The total transmission rate to and from the GCI is then approximately $10^{5 \mathrm{~N}}$.

$\begin{array}{llll}\text { Order of GCI } & \text { Entity } & \begin{array}{l}\text { Communicates with } \\ \text { Entities }\end{array} & \begin{array}{l}\text { Potential Capacity } \\ \text { (Rate and Scale) of } \\ \text { Communication }\end{array} \\ \text { Zeroth Order GCI } & \text { Individual Human } & \text { Individual Human } & 1 \\ \text { First Order GCI } & \text { Individual Human } & \text { Individual Human } & 1 \\ \text { First Order GCI } & \text { Individual Human } & \text { First Order AGI } & 1 \\ \text { First Order GCI } & \text { Individual Human } & \text { First Order GCI } & 1 \\ \text { First Order GCI } & \text { First Order AGI } & \text { First Order AGI } & 10^{5} \\ \text { First Order GCI } & \text { First Order AGI (N } & \text { First Order GCI } & 10^{5 \mathrm{~N}} \\ & \text { AGIs) } & \text { Individual Human } & 1 \\ \text { Second Order GCI } & \text { Individual Human } & \text { Second Order AGI } & 1 \\ \text { Second Order GCI } & \text { Individual Human } & \text { Second Order GCI } & 1 \\ \text { Second Order GCI } & \text { Individual Human } & \text { Second Order AGI (N } & 10^{5 \mathrm{~N}} \\ \text { Second Order GCI } & \text { Second Order AGI (N } & \text { AGIs) } & \\ & \text { AGIs) } & \text { First Order GCI (M } & 10^{5 \mathrm{MN}} \\ \text { Second Order GCI } & \text { First Order GCI (M } & \text { GCIs) } & \end{array}$

\section{GCI Based Communication Might be Perceived as Noise}

One of the characteristics a GCI is defined to have is the capacity to remove the barriers to massively scaling cooperation. For this reason, GCI based communication is likely to be very different than any current communication we're familiar with. Firstly, the capacity to dynamically form cooperation to optimize execution any process suggests that GCI based communication might require the capacity to spontaneously form networks in which participants cooperate to use the optimal choice of any available bandwidth and the optimal choice of any available protocol to achieve optimal fitness at executing whatever communication function is required. Rather than building a single device like a cell phone that uses a given protocol on a specific band, all cell phones, computers, IoT, and other devices might be built so they cooperate to use the best available protocol on the best available band to self-assemble the best available network.

The massive scale of peer to peer communication involved, as suggested by the table above, combined with the capacity to dynamically form cooperation to achieve optimal fitness at executing whatever communication function is required, and the potential capacity that communication might leverage complex interactions in order to reduce the communication required, togethers suggests that cooperation within a higher order civilization might potentially be too complex to be detected as communication. Any such transmission might be perceived as noise by a lower order civilization such as currently exists on earth.

\section{GCI Based Communication is Likely to be Wired not Wireless}

Though nature has demonstrated the ability of biological organisms to scale electrical transmission, as with electric eels, that transmission does not appear to be used to extend the complex communication that occurs within the organism to other organisms. Nature uses neural tissue as conduits for electrical impulses when communicating within the brain, and nature uses transmission through sound or other forms of energy when communicating at a distance, as opposed to simply scaling up the electrical signal and transmitting it. One reason might simply be to avoid interference with the transmitting brain's own thought processes. If so, then assuming evolution has explored a large number of potential 
solutions, the ability to massively scale communication on a planetary level without interference suggests that to avoid interference with lower order communication, communication at any given order of GCI is likely to be wired and not wireless.

\section{Identifying and Detecting Possible Modes of GCI Based Communication is a Higher Order Problem that Likely Requires GCI}

Functional modeling of problems and solutions defines problems or solutions as higher order when defining those problems, or when discovering those solutions, requires identifying $\mathrm{N}$ simultaneous interactions between the functions of the system, where $\mathrm{N}$ is higher than any human cognition has the capacity to reliably navigate, so that neither the problem nor solution can reliably be resolved, in which case one is likely to be solving the wrong problem with the wrong solution.

Identifying and detecting possible modes of communication within a civilization that has achieved GCI might be difficult if it is true that much of that communication might be wired. And for the above reasons, identifying and detecting possible modes of communication within or between civilizations that have achieved GCI is also potentially a problem of higher order complexity that is not reliably solvable without GCI or without another higher order intelligence such as an AGI. Since neither the problem nor the solution can reliably be resolved, by definition higher order problems are those in which one is likely to be trying to solve the wrong problem with the wrong solution. If so, then in trying to detect communication within or between civilizations that have reached the state of implementing a first order or higher order GCI, current efforts in the search for extraterrestrial intelligence (SETI) are likely to be looking in the entirely wrong direction.

\section{Detecting Higher Order Methods of Communication Used at a Distance}

Methods of communication that might be used by civilizations that have reached first order or higher GCI (higher order methods of communication) might however still have some definable characteristics that allow the search for such communication by a lower order civilization such as currently exists on earth. Firstly, according to the Kardashev scale, such civilizations might be expected to have an exponentially greater capacity to harness energy. This might locate such civilizations near to high energy sources. These sources might be black holes of a given mass, if as suggested in work by Penrose and Floyd [2], under certain conditions such black holes might be used to generate massive amounts of energy. Detecting such communication might involve looking for signatures of communication from near those bodies.

Higher order methods of communication at a distance might potentially be based on quantum communication or other mechanisms that are not transmitted through electromagnetic signals, and which therefore leverage transmission through some medium not currently known. Communication from any individual party might not be detectable by current methods, but communication with both parties might be correlated in some way. Detecting such correlations might involve identifying two such energy sources on opposite sides of the earth that might potentially have higher order civilizations nearby, and searching for such correlations in some kind of detectors on opposite sides of the earth. Exactly what those detectors would need to detect, and how to detect quantum communication through such correlations between such detection, is a matter for further investigation.

\section{Conclusions}

If the reasoning presented in this paper is correct, the conventional search for electromagnetic signals currently being performed in the search for extraterrestrial intelligence (SETI) might be looking for the wrong signature. If so, the approach to that search might benefit from being revised to reflect the concept of GCI. 


\section{References}

[1] Williams, Andy E. "Human Intelligence and General Collective Intelligence as Phase Changes in Animal Intelligence.” PsyArXiv, 11 July 2020. Web.

[2] PENROSE, R., FLOYD, R. Extraction of Rotational Energy from a Black Hole. Nature Physical Science 229, 177-179 (1971). https://doi.org/10.1038/physci229177a0 